\title{
AVALIAÇÃODOSHÁBITOS,CONHECIMENTOSEEXPECTATIVASDEALUNOSDEUM CURSODEPSICOLOGIA
}

\author{
Avaliação de alunos de psicologia
}

\author{
Makilim Nunes Baptista \\ Andréia Amadio $^{2}$ \\ Elen Carolina Rodrigues ${ }^{2}$ \\ Kívia Mendonça dos Santos ${ }^{2}$ \\ Silmara Aparecida Trindade Palludetti ${ }^{2}$
}

\section{Resumo}

\begin{abstract}
Esta pesquisa teve como objetivo analisar os hábitos acadêmicos, conhecimentos e expectativas de 101 alunos do $3^{\circ}$ e $4^{\circ}$ anos de um curso de Psicologia de uma Universidade particular. Foi utilizado um questionário contendo 55 questões das quais 28 foram analisadas neste artigo e aplicados coletivamente nas salas de aula. Os principais resultados demonstraram que a maioria dos alunos $68 \%$ do Grupo Total (GT) lê os textos antes das aulas; $51 \%$ gastam até 2 horas por semana com leituras extraclasse; $89 \%$ estudam antes das avaliações; 28\% sempre ou quase sempre questiona os professores durante a aula; $40 \%$ esclarece dúvidas em sala; $59 \%$ ( $3^{\circ}$ ano) e $76 \%$ ( $4^{\circ}$ ano) já tem linha teórica definida; $56 \%$ acha razoável a expectativa do mercado de trabalho e $82 \%$ pretende fazer pós-graduação. Estes dados devem ser considerados dentro do planejamento do curso a fim de proporcionar estratégias para acompanhar o desenvolvimento do aluno no decorrer do curso.
\end{abstract}

Palavras-chave: Universitários; Caracterização; Psicologia; Formação; Estudantes.

\section{Evaluation of Habits, Knowledge and Expectations of Psychology Students Evaluation of psychology students}

\begin{abstract}
The objective of this research was to analyze academic habits, knowledge and expectation of 101 students of which 3rd year and 4th year of Psychology course in a private university. It was used a questionnaire of 55 questions, 28 of witch were analyzed in this article, applied collectively in the classroom. The main results showed that the majority of the students $(68 \%)$ of the total group read the texts before the classes; $51 \%$ of the said they spend up to 2 hours per week on psychology reading outside the class; $89 \%$ study before the tests; $28 \%$ ask questions of the teachers; $40 \%$ clear up doubts with the teacher in the; $59 \%$ of 3rd year students and $76 \%$ of 4 th year students already have a defined theory line; $56 \%$ said that consider reasonable the current job market and $82 \%$ said they intend to go to postgraduate school. These data should be included in the planning of the course to development educational strategies to observe the student development.
\end{abstract}

Key Words: University students; Characterization; Psychology; Formation.

\section{INTRODUÇÃo}

É relevante conhecer as características dos alunos de um curso, pois, somente assim, o corpo docente e diretivo da instituição podem pensar em estratégias para isolar e eliminar variáveis negativas relacionadas à formação e expectativas dos alunos. Bem como podem preparar estratégias educacionais eficazes no desenvolvimento do seu currículo. Da mesma forma, as informações provenientes da caracterização do aluno que freqüenta um curso, como, por exemplo, seu poder econômico, hábitos, motivações e expectativas podem auxiliar na adaptação do

${ }^{1}$ Docente do Programa de Estudos Pós-Graduados em Psicologia da Universidade São Francisco - Itatiba/SP

${ }^{2}$ Psicólogas 
curso às necessidades e características deste alunado, bem como fornecer subsídios para desenvolvimento de programas que possam implementar habilidades acadêmicas importantes para sua formação.

Cosenza, Joly \& Primi (2003) afirmam que a avaliação de diversas variáveis educacionais pode ser considerada uma forma de acompanhar o desenvolvimento dos alunos durante a graduação. Além de ressaltar a necessidade de avaliações múltiplas que contemplem vários aspectos do conhecimento e diferentes habilidades. A fim de a instituição ter informações específicas para poder traçar um panorama de ações e aprimoramento de estratégias de ensino, confluentes com as diretrizes curriculares.

O conhecimento do alunado de um curso superior parece não ser preocupação constante das universidades, sendo que é necessário conhecer tais características para que a realidade do curso possa ser adequada à realidade do aluno (Campos, Silva-Filho, Campos \& Rocha, 1996a). Por exemplo, Bosi e Elias (2000) ressaltam, em seu trabalho, uma série de dificuldades e adaptações que os universitários recém-ingressos enfrentam nesta nova etapa da vida. Nesse mesmo sentido, é importante avaliar as dificuldades e/ou características dos estudantes de anos posteriores nos cursos universitários com o objetivo de acompanhar o desenvolvimento e adaptações às atividades pedagógicas. Favorecendo uma melhor adaptação do aluno ao curso e, automaticamente, diminuindo as chances de o aluno optar pela desistência de um curso universitário.

Grande parte das pesquisas sobre o psicólogo acaba se referindo a etapa de pós-formação, em que características da opção profissional são o objetivo principal (CRP, 1995). No entanto, nos últimos anos, parece haver um maior número de artigos que versam sobre questões referentes a levantamento das necessidades dos discentes (Dias, 1980; Bariani, 1995; Gomes \& cols. 1996). Até mesmo o provão deveria ter a função de detectar alguns problemas relacionados à formação do aluno, diagnosticando a realidade acadêmica, o que também acaba auxiliando em um diagnóstico geral mais abrangente desta realidade, apesar de algumas limitações deste tipo de avaliação levantadas por Primi, Landeira-Fernandez e Ziviani (2003).

Algumas pesquisas realizadas no Brasil, com o intuito de descrever hábitos de estudo de discentes, relatam resultados importantes para a reavaliação de metodologias no ensino. Por exemplo, Watanabe,
Cassertari, Santos, Lombard-Platet e Di Domenico (2001) pesquisaram 1.070 alunos de um curso de Psicologia de São Paulo, obtendo como um dos principais resultados a inadequação dos hábitos de estudo dos estudantes, sugerindo-se a necessidade de ações preventivas, especificamente relacionadas à elaboração de programas e estratégias que favoreçam reflexões do corpo docente sobre a didática e estratégias de ensino, bem como intervenções psicopedagógicas diferenciadas.

Carvalho (1986), avaliando a relação entre atividades extracurriculares de alunos de Psicologia (ex. estágios remunerados), conclui que estas auxiliam o discente no engajamento posterior na profissão. Sendo uma forma de aumentar a probabilidade de inserção profissional, revelando que os hábitos específicos dos alunos durante a graduação podem estar correlacionados com o seu aproveitamento pelo mercado de trabalho na Psicologia.

Silva e Botomé (1996), ao avaliarem a percepção de estudantes sobre o trabalho do psicólogo clínico, ressaltaram a influência da formação nas opiniões dos alunos. Indicaram que o curso de Psicologia acaba direcionando a percepção deles para determinadas opiniões sobre a própria psicologia, até mesmo a visão que o aluno terá de quem será este profissional e quais as suas possibilidades de atuação. Sendo assim, quanto maior a gama de informações e multiplicidades envolvidas no curso tanto mais os alunos poderão discriminar nuances da profissão e do mercado de trabalho.

Santos (1989), por meio da pesquisa com recémingressos no curso de Psicologia também avaliou as informações que estes tinham sobre as áreas de atuação. Concluiu que estas eram precárias e desorganizadas, sendo que a função social do psicólogo não chega a ser reconhecida. Demonstrando, mais uma vez, o quanto que a avaliação do aluno de Psicologia pode dar dicas de como promover estratégias para abarcar lacunas na formação e conhecimento dos alunos.

Por meio de análise de discurso de 53 estudantes de diversos cursos de uma universidade de Minas Gerais, Gondim (2002) levantou alguns aspectos. Tais aspectos apontam para a falta de definição do perfil profissional que o mercado de trabalho requisita, dificultando planos futuros, bem como a avaliação da qualidade dos estágios como insuficientes ou inadequados. Esses dados podem avaliar o quanto as universidades necessitam conhecer o alunado e desenvolver reordenações ou implementações nos serviços oferecidos. Não somente naqueles diretamente relacionados com o processo ensino- 
aprendizagem em sala de aula, mas também na relação entre o conteúdo programático, Lei de Diretrizes e Bases, estágios curriculares e extracurriculares e serviços de orientação na escolha de grade (quando possível), estágios, escolha de linhas teóricas e áreas de atuação e estratégias de inserção no mercado de trabalho.

Nos últimos anos, também se verificou o aumento de estatísticas relacionadas ao alunado das Universidades brasileiras, organizadas pelo Instituto Nacional de Estudos e Pesquisas Educacionais (Inep, 2002), por meio do Ministério da Educação e Cultura. Sendo que estes dados são considerados valiosos no sentido de proporcionarem, no âmbito nacional, algumas características socioeconômicas dos universitários.

Com base nas informações anteriores, o objetivo deste trabalho foi descrever características demográficas, sociais, econômicas e, principalmente, de formação dos alunos de um curso de Psicologia de uma Universidade particular.

\section{MÉTODO}

\section{Participantes}

Os participantes desta pesquisa foram 101 alunos do $3^{\circ}$ (51 alunos) e $4^{\circ}$ ano (50 alunos) do curso de Psicologia noturno, de uma Universidade do interior de São Paulo (região de Campinas), denominados consecutivamente de GA e GB. A média e o desvio-padrão das idades da amostra total foi de 24,7 anos (idade mínima 20 anos e idade máxima 53 anos; média de 21 anos). A amostra ainda se constituiu de $90 \%$ do sexo feminino e $10 \%$ do sexo masculino; $85 \%$ da amostra de solteiros; $86 \%$ residia com familiares; $75 \%$ cursou a maior parte (mais de $50 \%$ ) do ensino médio e fundamental em escola pública; $63 \%$ da amostra possuía atividade remunerada individual (75\% deste montante ganhavam até 4 salários mínimos); a renda familiar média comportou-se da seguinte forma: $21 \%$ até 5 salários mínimos, $39 \%$ de 6 a 10 salários, $20 \%$ de 11 a 15 salários, $10 \%$ de 16 a 20 salários e $10 \%$ acima de 20 salários.

\section{Material}

Para a coleta de informações foi utilizado um questionário contendo 55 questões tipo abertas e fechadas, desenvolvido pelo primeiro autor, das quais foram avaliadas 28 para este estudo, relacionadas a questões socioeconômicas, familiares, culturais e questões referentes à formação e hábitos acadêmicos. A última questão (tabela 5) do questionário foi baseada no estudo de Campos e cols. (1996a), com inclusões de autores e reordenação dos nomes ligados à ciência psicológica.

\section{Procedimento}

Os questionários foram aplicados coletivamente em sala de aula, com duração aproximada de 1 hora, pelos pesquisadores integrantes deste trabalho. A coleta dos dados ocorreu durante o período letivo de 2002 e, para tanto, foi solicitada a autorização da coordenação do Curso de Psicologia, bem como dos professores que ministravam aula no momento da aplicação do questionário.

Antes de os questionários serem entregues, os aplicadores verificaram, com o professor, a presença em sala de aula de, no mínimo, 70\% dos alunos regularmente matriculados, para o início da coleta dos dados. Os questionários foram entregues e os aplicadores explicaram os objetivos da pesquisa, além de informar aos participantes a não-obrigatoriedade da participação, instruindo-os para lerem e assinarem o Termo de Consentimento Livre e Esclarecido, antes de responder os questionários.

\section{Resultadose Discussão}

Os dados quantitativos obtidos foram analisados por meio de testes estatísticos não-paramétricos, adequados ao nível de mensuração de cada variável. Sendo que para variáveis nominais, foi aplicado o teste do Qui-quadrado, utilizando o nível de significância de 5\%, levando-se em consideração os dados brutos (Siegel, 1957), apesar de, em algumas respostas relatadas, aparecerem às porcentagens, somente como dados informativos, pois os testes sempre foram feitos com os dados brutos.

Como o objetivo deste estudo foi caracterizar parcialmente os alunos de uma Universidade particular da região de Campinas, é importante enfatizar que a metodologia utilizada neste trabalho tende a limitar as generalizações expostas à amostra estudada. Sendo que estes dados poderão auxiliar o corpo docente/ coordenação a conhecer melhor quem é o aluno desta instituição. Além de possibilitar o desenvolvimento de estratégias e metodologias de ensino adequadas às características desta população. 
Em relação à idade dos sujeitos e distribuição do gênero no curso, esta amostra se assemelhou à do estudo de Campos e cols. (1996a) que pesquisou uma subamostra de 98 alunos do $3^{\circ}$ e $4^{\circ}$ anos de uma Universidade particular no interior de São Paulo. No entanto, em relação ao número de sujeitos que cursou a maioria do ensino médio e fundamental em escola pública se verificou uma disparidade, já que, na amostra de Campos e cols. (1996a), a porcentagem foi inferior a este estudo, com 54\% dos alunos. Da mesma forma, a renda familiar também sugeriu diferença, sendo que na amostra de Campos e cols. (1996a) obteve-se menor porcentagem nas faixas de 6 a 10 salários $(27,6 \%$ vs. $39 \%)$ e maior porcentagem na faixa de 16 a 20 salários mínimos (18\% vs. 10\%). Baptista, Yoshimoto, Monelo e Baptista (1998) também encontraram, em uma amostra de 124 alunas de um curso de Psicologia de uma universidade particular, um predomínio de até cinco salários mínimos no primeiro, terceiro e quarto anos, referente à renda mensal, o que se aproxima dos resultados da presente pesquisa.

É importante citar que alguns dados relatados pela atual pesquisa não foram passíveis de comparação com outros estudos, devido à dificuldade em se encontrar bibliografias que pudessem ser comparadas aos dados atuais, como se pode observar nas questões relativas à freqüência de compra de livros exigidos pelos professores; leitura de textos antes da aula e o comportamento de estudo pré-avaliação. Mesmo assim, parece que estes dados são de suma importância para avaliar o perfil do estudante de Psicologia, já que tais indícios são fundamentais para explicar quais são os hábitos de estudo do alunado. Como as atividades extraclasse, segundo Carelli e Santos (1998), podem ser relacionadas a leituras de texto, resoluções de problemas, dentre outras atividades, não foi possível, pelo tipo de pergunta, avaliar se as leituras referenciadas eram relativas às obrigatórias para as provas ou leituras não necessariamente relacionadas com as avaliações.

Em relação à questão da frequiência de compra de livros exigidos pelos professores observou-se uma diferença estatística na variável $\left(\chi^{2}=23,8 ; \mathrm{gl}=2 ; \mathrm{p}=0,00\right)$ no GT. As categorias "nunca/quase nunca" (13\%) e "às vezes" (35\%) se diferenciaram da categoria "sempre/ quase sempre" (52\%). Apesar de as alternativas não especificarem uma quantidade específica e objetiva, percebeu-se que os alunos desta amostra regularmente adquirem os livros exigidos pelos professores, sendo que, provavelmente esta variável esteja ligada à condição econômica específica desta amostra. Da mesma forma, este indicativo deve ser visto com cautela, já que a compra de livros não necessariamente é sinônimo de estudo.

Na variável referente à frequiência de horas de estudo extraclasse que os alunos relataram realizar durante a semana, observou-se diferença estatisticamente significante nas respostas $\left(\chi^{2}=100,7 ; \mathrm{gl}=5 ; \mathrm{p}=0,00\right)$ no GT. A resposta "até duas horas" $(51 \%)$ prevaleceu dentre as demais $\left(\chi^{2}=12,1 ; \mathrm{gl}=5 ; \mathrm{p}=0,00\right)$, sendo assim distribuídas: "zero hora" (7\%); "até 4 horas" (22\%); "até 6 horas" (10\%); "até 8 horas" (7\%) e, "mais de 8 horas" (3\%). Estes resultados são diferentes dos encontrados na pesquisa do Inep (2002) referente ao ano de 2001, no qual $37,6 \%$ dos alunos estudam de 3 a 5 horas e $29,3 \%$ até duas horas, sendo que os alunos da presente amostra estudam menos tempo por semana do que a média nacional, no entanto não é especificado se este tempo é gasto com as leituras exigidas ou extraclasse.

Da mesma forma, em pesquisa realizada com os dados do Provão 2001 (Revista do provão, 2002), os melhores alunos de variados cursos relataram ter estudado, em média, 7 horas semanais, também não sendo especificado se estas horas se referiam a leituras exigidas ou não pelo corpo docente. Na pesquisa realizada por Carelli e Santos (1998), observou-se que os alunos de Psicologia noturnos são os que citam ter menor tempo médio de estudo semanal. Sendo uma média de estudo de $5,5 \mathrm{~h} / \mathrm{sem}$. Além do que, foi relatado, por estes autores, que uma das características que determinam a evolução e realização acadêmica é a ampliação do universo cultural do estudante, estando relacionado ao estudo extraclasse. Destaca-se a importância e a necessidade de os alunos dedicarem-se mais a esses períodos para garantir um reforço na aprendizagem, favorecendo uma formação acadêmica mais satisfatória.

Os dados da presente pesquisa também são dissonantes dos encontrados por Ferreira, Almeida e Soares (2001) que encontraram uma média de estudo dos universitários de seis horas por semana, subindo para doze horas nos períodos de exame. No entanto, os dados foram avaliados em um outro contexto cultural (Universidade do Minho), sendo necessária muita cautela para a comparação com a realidade brasileira.

$\mathrm{Na}$ questão relativa à leitura de textos antes das aulas constatou-se diferença estatística $\left(\chi^{2}=59,4 ; g l=2 ; p=0,00\right)$ no GT. Concluindo-se que o resultado mais apontado se relacionou à resposta "sempre/quase sempre" (68\%), quando comparado com as categorias "às vezes" (24\%) 
e "nunca/quase nunca" (8\%). Os alunos também relataram predominantemente estudar antes das avaliações com a categoria "sempre/quase sempre" (89\%), o que parece ser o esperado $\left(\chi^{2}=142,1 ; \mathrm{gl}=2\right.$; $\mathrm{p}=0,00$ ), quando comparado com as outras categorias "às vezes" (9\%) e "nunca/quase nunca" $(2 \%)$.

Em relação ao comportamento do aluno em estudar nos fins de semana, mesmo sem a presença de avaliações escolares, observou-se que os resultados não diferiram, demonstrando não haver prevalência de uma categoria, estando distribuídos de forma igualitária $\left(\chi^{2}=0,13 ; \mathrm{gl}=2 ; \mathrm{p}>0,05\right)$ no GT. Sendo "sempre/quase sempre" (33\%); "às vezes" (32\%) e "nunca/quase nunca" (35\%). Na pesquisa realizada por Carelli e Santos (1998), a maioria dos alunos de Psicologia do período noturno relatou estudar mais aos fins de semana, principalmente em detrimento da falta de tempo durante a semana, pois a maioria possuía a necessidade de se dedicar ao trabalho. Na mesma pesquisa, os autores ainda inferem que a quantidade de horas do fim de semana seria pouco para garantir um complemento adequado e necessário à formação profissional que se deseja.

Referente ao tempo dedicado aos estudos, especificamente a leitura de textos extras não solicitados pelos professores, observou-se que a resposta "às vezes" $(50 \%)$ se destacou dentre as outras respostas $\left(\chi^{2}=13,6\right.$; $\mathrm{gl}=2 ; \mathrm{p}=0,00)$ no GT. Ou seja, "sempre/quase sempre" (23\%) e "nunca/quase nunca" (27\%), sendo interessante notar que o horário disponível dos alunos pode estar diretamente relacionado com os comentários de Carelli e Santos (1998) sobre o tempo disponível, já que a falta de tempo também pode ser um agravante para a nãoleitura dos textos extras requisitado pelos docentes.

$\mathrm{O}$ resultado da pergunta referente à frequiência de questionamentos que os alunos fazem durante as aulas para os professores foi o da não-diferença estatística no GT. A distribuição das respostas ocorreu da seguinte forma "nunca/quase nunca" (29\%); "às vezes" $(43 \%)$ e "sempre/quase sempre" (28\%).

A respeito da questão sobre o esclarecimento de dúvidas em sala de aula, observou-se diferença estatisticamente significante $\left(\chi^{2}=8,3 ; \mathrm{gl}=2 ; \mathrm{p}=0,01\right)$ no GT. As respostas "nunca/quase nunca" $(20 \%)$ foram as menos apontadas quando comparadas com as categorias "às vezes" e "sempre/quase sempre" (ambas com 40\%).

Na pergunta referente à escolha de uma linha teórica, a análise estatística, por conveniência, foi realizada separadamente no grupo do terceiro e do quarto ano $(\mathrm{GA}=21$ respostas negativas e $\mathrm{GB}=12)$. Observou-se que no GA não houve diferença estatística nas respostas $\left(\chi^{2}=1,5 ; \mathrm{gl}=1 ; \mathrm{p}>0,05\right)$. No entanto este resultado não se repetiu no grupo do quarto ano $\left(\chi^{2}=13,5 ; \mathrm{gl}=1\right.$; $\mathrm{p}=0,00)$. Apesar de serem duas amostras diferentes, pode-se hipotetizar que o aluno do quarto ano terá de se definir por uma linha teórica, já que os estágios e a necessidade de opção, decorrente do quinto ano, estão mais próximos. Campos, Souza, Catão e Campos (1996b) relatam que a escolha da linha teórica faz parte de um dos principais dilemas que o aluno de psicologia enfrenta em sua formação. Já que esta também acaba sendo influenciada por características pessoais dos professores. Os mesmos autores concluíram que $20 \%$ dos alunos responderam que houve uma "razoável" influência percebida da relação positiva entre professor e sujeito na escolha da abordagem.

Referente ao tipo de preferência por linha teórica (tabela 1) concluiu-se pela diferença estatisticamente significante entre as respostas $\left(\chi^{2}=52,6 ; \mathrm{gl}=4 ; \mathrm{p}=0,00\right)$, constatando-se que o bloco de comportamental e respostas em branco se diferenciou da psicanálise $\left(\chi^{2}=6,0 ; \mathrm{gl}=2\right.$; $\mathrm{p}=0,05)$. É interessante notar que as respostas em branco correspondem a aproximadamente $1 / 3$ das respostas totais e são mais freqüentes no terceiro ano. Estes resultados não confirmam os achados por Campos e cols. (1996b),

Tabela 1: Distribuição da linha teórica de preferência.

\begin{tabular}{lcccccc}
\hline \multirow{1}{*}{ RESPOSTAS } & \multicolumn{2}{c}{ GA } & \multicolumn{2}{c}{ GB } & \multicolumn{2}{c}{ GT } \\
\cline { 2 - 7 } & $N$ & $\%$ & $N$ & $\%$ & $N$ & $\%$ \\
Psicanálise & 7 & 14,0 & 13 & 26,0 & 20 & 20,0 \\
Comportamental & 20 & 39,0 & 18 & 36,0 & 38 & 37,0 \\
Existencial & 0 & 0 & 3 & 6,0 & 3 & 3,0 \\
Comportamental-Cognitiva & 3 & 6,0 & 2 & 4,0 & 5 & 5,0 \\
Respostas em branco & 21 & 41,0 & 14 & 28,0 & 35 & 35,0 \\
Total & 51 & 100,0 & 50 & 100,0 & 101 & 100,0 \\
\hline
\end{tabular}


que encontraram preferência dos alunos pela Psicanálise. No entanto é importante salientar que estas escolhas podem estar diretamente relacionadas com a constituição do corpo docente e a orientação da coordenação do curso, que pode ter influência na contratação dos docentes e suas linhas teóricas específicas.

A tabela 2 expressa a preferência dos alunos para as áreas de atuação, observando-se novamente dife- mento com diversas Agências Formadoras, uma tendência das opções de estágio ainda serem na área clínica, o que corrobora os dados da presente pesquisa.

Em relação às atividades extracurriculares em que os alunos já participaram, observaram-se os seguintes resultados: não houve diferença estatística na categoria congresso $\left(\chi^{2}=2,9 ; g l=1 ; p>0,05\right)$. Houve diferença nas outras categorias, sendo palestras $\left(\chi^{2}=77,4 ; \mathrm{gl}=1\right.$;

Tabela 2: Apresentação da preferência de área de atuação.

\begin{tabular}{lcccccc}
\multicolumn{1}{c}{ RESPOSTAS } & \multicolumn{2}{c}{ GA } & \multicolumn{2}{c}{ GB } & \multicolumn{2}{c}{ GT } \\
\cline { 2 - 6 } Clínica & $N$ & $\%$ & $N$ & $\%$ & $N$ & $\%$ \\
Escolar & 15 & 29,0 & 17 & 34,0 & 32 & 31,0 \\
Trabalho & 7 & 14,0 & 2 & 4,0 & 9 & 9,0 \\
Esporte & 8 & 16,0 & 7 & 14,0 & 15 & 14,0 \\
Saúde & 1 & 2,0 & 1 & 2,0 & 2 & 2,0 \\
Consumidor/Marketing & 12 & 23,0 & 19 & 38,0 & 31 & 31,0 \\
Social & 0 & 0 & 1 & 2,0 & 1 & 1,0 \\
Jurídica/Forense & 2 & 4,0 & 0 & 0 & 2 & 2,0 \\
Não me defini & 1 & 2,0 & 2 & 4,0 & 3 & 3,0 \\
Respostas em branco & 1 & 2,0 & 1 & 2,0 & 2 & 2,0 \\
Total & 4 & 8,0 & 0 & 0 & 4 & 5,0 \\
\hline
\end{tabular}

rença estatisticamente significante $\left(\chi^{2}=129,5 ; \mathrm{gl}=9\right.$; $\mathrm{p}=0,00)$. As respostas mais votadas foram "clínica" e "saúde", quando comparadas com o próximo conjunto de categorias, ou seja, "trabalho" e "escolar" $\left(\chi^{2}=7,0\right.$; $\mathrm{gl}=2 ; \mathrm{p}=0,03)$, diferenciando-se da categoria "jurídica/ forense" $\left(\chi^{2}=6,5 ; \mathrm{gl}=2 ; \mathrm{p}=0,04\right)$.

As opções mais apontadas ainda se referem às áreas clássicas da Psicologia, como observado, há mais de 25 anos, por Mello (1977). Exceto a área da saúde (que também pode ser considerada clínica, porém emergente), que, segundo Lo Bianco, Bastos, Nunes e Silva (1994), Mello (1996) e Yamamoto, Oliveira, Siqueira e Carvalho (1997), pode ser considerada uma tendência na atualidade, principalmente decorrente das Ações Integradas de Saúde (AIS) datada de 1983, aumentando as chances da inserção do psicólogo na área da saúde. Carvalho (1984) também aponta a expansão de áreas do trabalho dos psicólogos brasileiros, bem como discute a questão da identidade profissional, já que, em campos emergentes, nem sempre se observa uma preparação adequada da formação pelas instituições de ensino. De forma mais específica, Gonçalves (1999) aponta, em uma pesquisa de levanta- $\mathrm{p}=0,00) ; \operatorname{cursos}\left(\chi^{2}=44,4 ; \mathrm{gl}=1 ; \mathrm{p}=0,00\right)$ e estágios $\left(\chi^{2}=6,0 ; \mathrm{gl}=1 ; \mathrm{p}=0,01\right)$ apontados de forma decrescente de frequiência. Sendo assim, a quantidade de respostas para congressos não demonstrou diferença estatística, o que denota uma igualdade entre participar e não participar de congresso (respostas negativas $=57 \mathrm{x}$ respostas positivas=40). A não-participação em estágios foi maior do que a participação (respostas negativas $=60$ $\mathrm{x}$ respostas positivas $=36$ ), talvez também porque a oferta de estágios no mercado de trabalho seja menor do que a oferta de congressos. Já na categoria palestras (respostas negativas $=6 \times$ respostas positivas $=94)$ e cursos (respostas negativas $=16 \mathrm{x}$ respostas positivas $=82$ ), percebe-se uma maior freqüência de respostas em prol da participação destes eventos.

Ainda relacionado à participação do aluno em atividades extracurriculares, pode-se afirmar que tais atividades são fundamentais para aumentar os conhecimentos dos alunos em relação ao seu futuro campo de atuação e seu engajamento em atividades profissionais posteriores. Carvalho (1986) aponta para uma relação entre atividades extracurriculares desempenhadas por 
alunos de psicologia e maior engajamento no mercado profissional. Principalmente relacionado a estágios, sendo que aproximadamente $30 \%$ da amostra estudada pela autora engajou-se em atividade profissional por meio de estágios extracurriculares. Há também de se considerar que a oferta de estágios extracurriculares para alunos de graduação parece não ser adequado à demanda, como afirmam Campos e cols. (1996b). Da mesma forma, Andrade (1998) aponta que uma das funções de um curso de Psicologia seria favorecer um aprendizado relacionado com o desenvolvimento de um potencial científico no aluno e, uma das formas de se incentivar tal característica seria por intermédio de atividades extracurriculares, principalmente congressos científicos.

Referente às expectativas dos alunos sobre a situação do mercado de trabalho futuro (péssimo, ruim, razoável, bom), observou-se a predominância da categoria "razoável" $\left(\chi^{2}=80,9 ; \mathrm{gl}=3 ; \mathrm{p}=0,00\right)$. Os resultados aproximaram-se dos relatados por Campos e cols. (1996a), que ressaltam a tendência de sua amostra de um grupo de alunos noturnos de Psicologia indicarem a categoria "razoável" e Silva e Campos (1992), por meio de uma amostra de 270 alunos recém- ingressos em um curso de Psicologia também aponta uma predominância na resposta "regular".

Como aponta os estudos do CRP (1995), grande parte dos alunos que se formam ao menos chega a atuar na área da Psicologia. Além do que, atualmente, o cenário profissional não se mostra receptivo, já que nas últimas décadas os cursos de Psicologia se proliferaram de maneira descontrolada. Ainda segundo Duran (1994) e Bastos e Achcar (1994), as condições econômicas e políticas nacionais acabam refletindo na profissão do Psicólogo de maneira negativa, gerando um empobrecimento da classe.

A amostra respondeu a questão da intencionalidade de execução de pós-graduação de forma tendenciosa para a afirmação $\left(\chi^{2}=112,2 ; \mathrm{gl}=2 ; \mathrm{p}=0,00\right)$, sendo predominante tal intenção. É interessante assinalar a alta porcentagem de alunos que deseja continuar seus estudos, provavelmente pela percepção de um mercado de trabalho mais competitivo por meio das transformações políticas, econômicas e sociais, como apontam Bastos e Achcar (1994).

Apesar de a pergunta da presente pesquisa não especificar o tipo de pós-graduação desejada (lato ou stricto sensu), além de ser realizada com alunos, somente 30,81\% de psicólogos haviam realizado algum tipo de pósgraduação segundo o CRP (1995), observando-se uma alta expectativa da presente amostra. Talvez esta expectativa esteja relacionada com as novas exigências que o Instituto Nacional de Estudos e Pesquisas Educacionais vem fazendo na área acadêmica, podendo este fenômeno estar influenciando, direta ou indiretamente o alunado para um novo mercado de trabalho.

Um outro fator que deve ser considerado, como apontam Lo Bianco e cols. (1994) e Natário (1999), se refere ao aumento da necessidade da continuidade da graduação. Bem como as novas condições de pesquisa que algumas universidades vêm proporcionando, o que pode estar refletindo diretamente no desejo de realização de uma pós-graduação.

A expectativa de salário (tabela 3 ) também se mostrou estatisticamente diferente $\left(\chi^{2}=78,2 ; \mathrm{gl}=5 ; \mathrm{p}=0,00\right)$. As categorias mais apontadas foram "mais de 3 salários" e "mais de 6 salários", se diferenciando da próxima categoria "mais de 9 salários" $\left(\chi^{2}=18,5 ; \mathrm{gl}=2 ; \mathrm{p}=0,00\right)$. Em relação a este ponto, apesar da limitação da amostra, pode-se considerar que a perspectiva de ganho salarial não parece ser tão irreal quando comparada com a pesquisa realizada com profissionais, realizadas pelo CRP (1995), na qual $31,49 \%$ dos formados tinham um ganho de até 5 salários mínimos. No entanto, deve-se ter precauções com esta comparação, já que a amostra adotada pelo CRP possuía

Tabela 3: Frequiência e porcentagem de quanto se espera ganhar como psicólogo após sua formação.

\section{RESPOSTAS}

Até 3 salários

Mais de 3 salários

Mais de 6 salários

Mais de 9 salários

Mais de 12 salários

Respostas em branco

Total
GA

$N$
2
15
27
4
3
0
51

GB

\begin{tabular}{cccccc}
$N$ & $\%$ & $N$ & $\%$ & $N$ & $\%$ \\
2 & 4,0 & 3 & 6,0 & 5 & 5,0 \\
15 & 29,0 & 15 & 30,0 & 30 & 30,0 \\
27 & 53,0 & 16 & 32,0 & 43 & 42,0 \\
4 & 8,0 & 7 & 14,0 & 11 & 11,0 \\
3 & 6,0 & 8 & 16,0 & 11 & 11,0 \\
0 & 0 & 1 & 2,0 & 1 & 1,0 \\
& 100,0 & 50 & 100,0 & 101 & 100,0 \\
\hline
\end{tabular}


profissionais já formados e com experiência, podendo haver um viés, no sentido destes profissionais já terem experiência no mercado de trabalho, pós-graduação e outras variáveis confundidoras.

A tabela 4 demonstra a opinião dos alunos em relação a qual área pertence a Psicologia, observando-se diferença estatística nos seguintes resultados: biologia $\left(\chi^{2}=16,0 ; \mathrm{gl}=1 ; \mathrm{p}=0,00\right)$; parapsicologia $\left(\chi^{2}=19,4 ; \mathrm{gl}=1 ; \mathrm{p}=0,00\right)$; ciências humanas (não é possível realizar a prova); religioso $\left(\chi^{2}=70,5 ; \mathrm{gl}=1\right.$; $\mathrm{p}=0,00)$; ciências exatas $\left(\chi^{2}=64,0 ; \mathrm{gl}=1 ; \mathrm{p}=0,00\right)$; não ciência $\left(\chi^{2}=49,0 ; g l=1 ; p=0,00\right)$. Chamam a atenção as respostas relacionadas à concordância da Psicologia para com parapsicologia, religioso, ciência exata e não ciência, em que alguns alunos apontam para uma relação equivocada. Estes dados não corroboram os resultados de Silva e Campos (1992), que encontraram em alunos de primeiro ano a predominância de respostas relacionadas às áreas humanas, biológicas e/ou ambas. No entanto, os autores não relataram ter dado outras opções como na pergunta
Uma análise mais específica mostra que a diferença estatística começa a partir de São Tomás de Aquino, ou seja, Skinner, Piaget, Freud, Watson, Jung, Rogers, Sartre, Reich, Marx, Strauss $\left(\chi^{2}=41,3\right.$; $\mathrm{gl}=10 ; \mathrm{p}=0,00)$ formariam o primeiro bloco de nomes. Estes resultados se aproximaram dos encontrados por Campos e cols. (1996a), com exceção de Watson, que não continha no trabalho original citado. Um dado interessante se referiu às respostas de nomes não relacionados à Psicologia, como, por exemplo, Green Smiths, Gandhi, Stravinsky, Alan Kardec, Vallosty, Drummond e Paulo Coelho, apontando o desconhecimento de uma pequena parcela da amostra em relação aos nomes ligados à Psicologia. Figueiredo (1983) aponta para o fato de que muitos alunos trazem consigo aspirações incompatíveis com a prática científica, também denominada de concepções anticientíficas, em que se observa incompatibilidade entre estas visões e o exercício profissional responsável e bem fundamentado da Psicologia.

Tabela 4: Distribuição das respostas de como a psicologia é considerada.

\begin{tabular}{lcc}
\multicolumn{1}{c}{ RESPOSTAS } & \multicolumn{2}{c}{ GT } \\
\cline { 2 - 3 } & CONCORDO & DISCORDO \\
Biologia & $\%$ & $\%$ \\
Parapsicologia & 70 & 30 \\
Ciência humana & 28 & 72 \\
Religioso & 100 & 0 \\
Ciência exata & 8 & 92 \\
Não ciência & 10 & 90 \\
Total & 15 & 85 \\
& 231 & 369 \\
\hline
\end{tabular}

da presente pesquisa (ex. religião, ciência exata), o que pode se caracterizar em um viés de resposta.

Na tabela 5 é expressa a opinião dos alunos sobre quais os nomes ligados à ciência psicológica. A lista possuía nomes realmente relacionados à Psicologia, nomes não relacionados e nomes inventados, a fim de realmente avaliar o conhecimento dos alunos sobre os principais autores, baseado no estudo de Campos e cols. (1996a). Observou-se uma diferença estatística entre as respostas $\left(\chi^{2}=915,4,0 ; \mathrm{gl}=23\right.$; $\mathrm{p}=0,00)$, não sendo consideradas as dimensões que não tiveram alguma resposta.

\section{CONSIDERAç̃óes Finals}

É de suma importância conhecer as características dos alunos de uma Universidade. As variáveis econômicas, comportamentos de estudo, motivações e aspectos sociais/ culturais podem influenciar de sobremaneira no desempenho e engajamento profissional destes.

Pode-se observar nesta amostra alguns hábitos de estudo inadequados ou insuficientes. Com isso acaba sinalizando, sem haver a intenção de generalização para outras amostras, a necessidade de projetos específicos 
Tabela 5: Assinalar os principais nomes ligados à ciência da psicologia.

\begin{tabular}{|c|c|c|c|c|c|c|}
\hline \multirow[t]{2}{*}{ RESPOSTAS } & \multicolumn{2}{|c|}{ GA } & \multicolumn{2}{|c|}{ GB } & \multicolumn{2}{|c|}{ GT } \\
\hline & $N$ & $\%$ & $N$ & $\%$ & $N$ & $\%$ \\
\hline Stravinsky & 5 & 10,0 & 0 & 0 & 5 & 4,9 \\
\hline F. Pearls & 4 & 8,0 & 9 & 18,0 & 13 & 12,8 \\
\hline Jean Piaget & 51 & 100,0 & 43 & 86,0 & 94 & 93,0 \\
\hline Jorge Amado & 0 & 0 & 3 & 6,0 & 0 & 0 \\
\hline Madre Tereza de Calcutá & 0 & 0 & 0 & 0 & 0 & 0 \\
\hline Carl Rogers & 42 & 82,0 & 44 & 88,0 & 86 & 85,1 \\
\hline John Watson & 43 & 84,0 & 45 & 90,0 & 88 & 87,1 \\
\hline Chico Chavier & 0 & 0 & 2 & 4,0 & 2 & 1,9 \\
\hline Jean P. Sartre & 40 & 78,0 & 43 & 86,0 & 83 & 82,1 \\
\hline Alan Kardec & 1 & 2,0 & 3 & 6,0 & 4 & 3,9 \\
\hline Içami Tiba & 0 & 0 & 2 & 4,0 & 2 & 1,9 \\
\hline São Tomás de Aquino & 17 & 33,0 & 20 & 40,0 & 37 & 36,6 \\
\hline Sigmund Freud & 48 & 94,0 & 45 & 90,0 & 93 & 92,0 \\
\hline Green Smiths & 4 & 8,0 & 8 & 16,0 & 12 & 11,8 \\
\hline Karl Marx & 34 & 67,0 & 31 & 62,0 & 65 & 64,3 \\
\hline B. F. Skinner & 50 & 98,0 & 49 & 98,0 & 99 & 98,0 \\
\hline Carlos Drummond & 0 & 0 & 3 & 6,0 & 3 & 2,97 \\
\hline C. Levi - Strauss & 36 & 70,0 & 27 & 54,0 & 63 & 62,3 \\
\hline M. Gandhi & 8 & 16,0 & 3 & 6,0 & 11 & 10,8 \\
\hline João Paulo II & 0 & 0 & 0 & 0 & 0 & 0 \\
\hline Victor Hugo & 0 & 0 & 0 & 0 & 0 & 0 \\
\hline Carl Jung & 42 & 82,0 & 45 & 90,0 & 87 & 86,1 \\
\hline Aaron Beck & 3 & 6,0 & 5 & 10,0 & 8 & 7,9 \\
\hline Gabriel G. Marques & 1 & 2,0 & 0 & 0 & 1 & 0,9 \\
\hline Wilhelm Reich & 33 & 65,0 & 42 & 84,0 & 75 & 74,2 \\
\hline Paulo Coelho & 1 & 2,0 & 1 & 2,0 & 2 & 1,9 \\
\hline D. Vallosty & 6 & 12,0 & 0 & 0 & 6 & 5,9 \\
\hline Artur Moreira Lima & 1 & 2,0 & 0 & 0 & 1 & 0,9 \\
\hline
\end{tabular}

no desenvolvimento da complementação do número de horas em leituras extraclasse semanais. Não somente diretamente relacionadas com o material exigido pelo corpo docente, mas também de leituras associadas aos conteúdos ministrados e obrigatórios. Além deste ponto importante, maior é a necessidade de se conhecer como é aproveitado o tempo que é dedicado ao estudo, como, por exemplo quais as estratégias de estudo destes.

Mesmo que a própria universidade não consiga oferecer um número razoável de eventos internos, é de suma importância que o corpo docente seja também orientado para fornecer informações e incentivar a participação (ou como acessá-las, como por exemplo as páginas de eventos do CRP, CFP, SBP, etc.) dos alunos em diversos eventos (estágios, cursos, congressos, simpósios, dentre outros) que ocorrem durante a formação. Desta maneira, o aluno teria, pelo menos, a possibilidade de aumentar seus conhecimentos técnicosteóricos e formar um arcabouço de conhecimentos que o auxiliarão na escolha de áreas de atuação e linha teórica ao final da sua formação.

Silva (2000) afirma que a vida acadêmica pode ser influenciada, direta ou indiretamente, pelo corpo de conhecimento que o aluno traz antes mesmo do ingresso a um curso universitário, a exemplo de suas expectativas, leituras anteriores do curso, planos profissionais futuros, informações e preconceitos. Sendo assim, se este corpo de conhecimento está distorcido 
(ex: acreditar que Parapsicologia e Psicologia são sinônimos), é de suma importância que este equívoco seja elucidado em sala de aula ou por meio de palestras, simpósios, programas científicos, dentre outros. Nesta direção, Bosi e Elias (2000) relatam a importância de se conhecer os aspectos que determinam a escolha do aluno ao curso, já que parece haver

\section{REFERÊNCIAS}

Andrade, M. C. M. (1998). O ato de investigar e a produção de conhecimento. Cadernos de Psicologia, 5, 63-66.

Baptista, M. N., Yoshimoto, L.W., Monelo, M. R., Baptista, R. A.W., \& Berti, A. A. (1998). Nível e fontes de estresse em alunos de psicologia. PSICO-USF, 3, 61-76.

Bariani, I. C.D. (1995). Um estudo exploratório sobre os motivose os significados associados à prática da pesquisa em estudantes universitários. Estudos de Psicologia, 12, 57-63.

Bastos, B. V. A., \& Achcar, R. (1994). Dinâmica profissional e formação do psicólogo: uma perspectiva de integração. Em Conselho Federal de Psicologia. Psicólogo brasileiro - práticas emergentes e desafios para a formação. São Paulo: Casa do Psicólogo.

Bosi, M. L. M., \& Elias, T. F. (2000). Um novo caminho: perfil e trajetórias de alunos de Psicologia ingressos como portadores de diploma. Estudos de Psicologia - PUC Campinas, 17 (2), 31-40.

Campos, L. F. L., Silva-Filho, N., Campos, P. R., \& Rocha, R. L. (1996a). Caracterização dos Alunos de Psicologia da USF/ Itatiba: Características, Opiniões e Expectativas. PSICOUSF, 1 (2), 57-82.

Campos, L. F. L., Souza, K.C. C., Catão, E. C., \& Campos, P. R. (1996b). Fatores motivacionais na escolha de abordagens teóricas em psicologia clínica. Estudos de Psicologia, 13(1), 41-54.

Carelli, M. J. G., \& Santos, A. A. A. (1998). Condições temporais e pessoais de estudo em universitários. Psicologia Escolar e Educacional, 2, 265-278.

Carvalho, A. M. A. (1984). Modalidades alternativas de trabalho para psicólogos recém-formados. Cadernos de análise do comportamento, 6, 1-14.

Carvalho, A.M.A. (1986). Formação profissional e atuação do psicólogo: alguns dados a respeito de relações entre atividades extracurriculares desempenhadas por alunos de psicologia e condições de atuação após a formatura. Boletim de Psicologia, 36 (85), 31-39. diversas variáveis que incidem sobre tal escolha. Sendo assim, de posse destas informações, coordenadores e professores podem desenvolver estratégias específicas no aperfeiçoamento do aluno ao curso de graduação, sendo tais características fundamentais para a implantação de trabalhos psicopedagógicos pelas universidades.

Conselho Regional de Psicologia - CRP-06. (1995). Psicologia: formação, atuação profissional e mercado de trabalho. (São Paulo, Mato Grosso e Mato Grosso do Sul).

Cosenza, R.C., Joly, M.C.R.A., \& Primi, R. (2003). Perspectivas de Avaliação no Ensino Superior: um estudo sobre o Exame Nacional de Cursos de Administração. Avaliação, 8, 155-172.

Dias, M. L. (1980). O motivo de realização entre os estudantes do curso de formação de psicólogos da PUC/RS. PSICO, 17, 81-93.

Duran, A.P. (1994). Alguns dilemas na formação do psicólogo: buscando sugestões para superá-los. Em Conselho Federal de Psicologia. Psicólogo brasileiro - práticas emergentes e desafios para a formação. São Paulo: Casa do Psicólogo.

Ferreira, J. A., Almeida, L. S., \& Soares, A. P. (2001). Adaptação acadêmica em estudantes do $1^{\circ}$ ano: diferenças de gênero, situação de estudante e curso. PSICO-USF, 6 (1), 1-10.

Figueiredo, L. C. M. (1983). Notas impopulares sobre a formação do Psicólogo. Cadernos de Análise do Comportamento, 4, 1-16.

Gomes, W. B., Teixeira, M. A. P., Crescente, D. B., Fachel, J., Sehn, L., \& Klarmann, P. (1996). Atitudes e crenças de estudantes universitários sobre psicoterapia e psicólogos. Psicologia: Teoria e Pesquisa, 2(12), 121 - 127.

Gonçalves, C. L. C. (1999). Formação geral e estágio acadêmico em psicologia escolar. Em C. Witter. (Org)., Ensino de psicologia (pp.143-168). Campinas: Editora Alínea.

Gondim, S. M. G. (2002). Perfil profissional e mercado de trabalho: relação com a formação acadêmica pela perspectiva de estudantes universitários. Estudos de Psicologia, 7 (2), 299-309.

Instituto Nacional de Estudos e Pesquisas Educacionais INEP. [on line]. Acesso em 11 agosto de 2002. Disponível em www.inep.gov.br.

Lo Bianco, A. C., Bastos, A. V. B., Nunes, M. L. T., \& Silva, R. C. (1994). Concepções e atividades emergentes na psicolo- 
gia clínica: implicações para a formação. Em Conselho Regional de Psicologia. Psicólogo Brasileiro - práticas emergentes e desafios para a formação. São Paulo: Casa do Psicólogo.

Mello, S. L. (1977). As atividades profissionais dos psicólogos: ensino e aplicações da psicologia. Em S. L. Mello (Org.), Psicologia e Profissão em São Paulo (pp.15-61). São Paulo. Editora Ática.

Mello, S. L. (1996). Formação do Psicólogo. Cadernos de Psicologia, 4, (5), 11-17.

Natário, E. G. (1999). Atividade de pesquisa em um curso de psicologia. Em C. Witter. (Org), Ensino de psicologia (pp. 97-117). Campinas: Editora Alínea.

Primi, R., Landeira-Fernandez, J., \& Ziviani, C. (2003). O Provão de Psicologia; objetivos, problemas, conseqüências e sugestões. Psicologia: Teoria e Pesquisa, 19, 109-116.

Revista do Provão. (2002). Quem é quem: os melhores do provão 2001. Brasília, 7, 7, pp.5-6.

Santos, M. A. (1989). O Psicólogo: atuação profissional e função social segundo a percepção de estudantes de Psicologia. Estudos de Psicologia, 6, 5-30.

Siegel, S. (1957). Non-parametric statistics for behavioral sciences. New York: McGraw-Hill.

\section{Endereço para correspondência:}

Makilim Nunes Baptista

Rua: Dr. Miguel Pierrô, 61

CEP: 13083-300 - Campinas, SP

e-mail: daherbaptista@ig.com.br

Andréia Amádio de Oliveira

Rua 24 de maio, 235 - Centro

CEP: 13330-060 - Indaiatuba, SP

e-mail: amadio.oliveira@bol.com.br

Elen Carolina Rodrigues

Rua Ferdinando Delamain, 178 - Centro

CEP: 13600-073 - Araras, SP

e-mail: ec.Rodrigues@bol.com.br
Silva, L. B. C. (2000). Representação social da psicologia enquanto ciência e profissão em alunos do primeiro semestre do curso de psicologia da Universidade São Marcos. Interações, 5, 111-144.

Silva, G. G., \& Campos, L. F. L. (1992). Caracterização dos alunos ingressantes em dois cursos recém-abertos de psicologia: um estudo comparativo sobre suas características, opiniões e expectativas. Estudos de Psicologia, 9, 92 - 110.

Silva, V. L. M., \& Botomé, S.P. (1996). Situações e locais de atuação do psicólogo clínico na percepção de estudantes de Psicologia. Psicologia (Sociedade de Estudos Psicológicos), 3, 11-34.

Yamamoto, O. H., Oliveira, S. C. C., Siqueira, G. S., \& Carvalho, D. B. (1997). Psicologia e escolha profissional: a imagem da profissão no RN. PSICO. Porto Alegre, 28, 17-33.

Watanabe, O. M., Cassertari, L., Santos, M. L. M., LombardPlatet, V. L. V., \& Di Domenico, V. G. C. (2001). Um levantamento dos hábitos de estudo dos alunos do Curso de Psicologia do Centro Universitário FMU. Psikhe, 6, 60-7.

Recebido em: 23/04/04

Revisado em: 13/05/04

Aprovado em: 17/06/04

Kívia Mendonça dos Santos

Rua Basílio Antonio Fernandes, 241

CEP: 13160-000 - Artur Nogueira, SP

e-mail:kiviapsico@yahoo.com.br

Silmara Ap. Trindade Palludetti

Rua Sebastião Camargo Schimidt, 280

CEP: 13600-000 - Araras, SP 